Hautarzt 2017 · 68:987-993

https://doi.org/10.1007/s00105-017-4054-9

Online publiziert: 9. Oktober 2017

(c) Der/die Autor(en) 2017. Dieser Artikel ist eine Open-Access-Publikation.

CrossMark

L. Rüther · L. Bolke · G. R. Schlippe - W. A. Voss

Münster, Deutschland

\title{
Hyaluronan als Schlüssel zur schnelleren Wundheilung in humanen 3-D-Vollhautmodellen
}

thelzellen an [5]. Fibroblasten produzieren Kollagenvorstufen zur Bildung eines Granulationsgewebes, um der Wunde Festigkeit zu verleihen. Das Granulationsgewebe ist reich an Hyaluronan, das durch die Fähigkeit das 3000-Fache des Eigengewichtes an Feuchtigkeit absorbieren $\mathrm{zu}$ können, eine intensiv hydrierte Matrix bietet [12]. Mit fortlaufender Wundheilung beginnen die Kollagenfasern zu schrumpfen, ziehen dadurch die Wundränder zueinander, sodass sich die Wundoberfläche verkleinert [14]. In der Regenerationsphase wird die Wunde durch die Teilung und Migration von Keratinozyten verschlossen (Reepithelisierung). Diesem Prozess kommt bei der Wundheilung eine entscheidende Bedeutung zu mit dem Ziel, die natürliche Barrierefunktion der Haut wiederherzustellen [16]. Hierbei spielt $\mathrm{HA}$, das im Menschen ubiquitär vorkommt, sich aber zu etwa $50 \%$ in der Haut befindet, eine entscheidende Rolle. So wurde bereits beschrieben, dass HA in die Regulation der Proliferation von Basalkeratinozyten involviert ist [2] und mittels des Oberflächenrezeptors CD44 deren Migration vermittelt [8].

In der vorliegenden Studie wurden 3-D-Vollhautmodelle verwendet, die aus primären humanen Keratinozyten und Fibroblasten $\mathrm{zu}$ einem funktionellen
Multilayer rekonstruiert werden. Diese Modelle weisen eine, verglichen mit nativer menschlicher Haut, ähnliche Wachstumscharakteristik, ein analoges Expressionsmuster sowie eine vergleichbare Barrierefunktion auf [6,7], wodurch sie sich sehr gut für Wundheilungsstudien [15] eignen.

Ziel der Studie war es, die Wundheilungsgeschwindigkeit durch Messen der Reepithelisierungskinetikzu analysieren. Hierzu wurden die mittels einer $4 \mathrm{~mm}$ Biopsiestanze verwundeten Vollhautmodelle 2-mal täglich mit $10 \mu \mathrm{l}$ Viscontour ${ }^{\circledR}$ SERUM MED, das -verglichen mit anderen Produkten - ausschließlich Natriumhyaluronat (NHA) als Wirkstoff enthält, behandelt. Durch den Nachweis und die Quantifizierung der Entzündungsmarker IL-1 $\alpha$ und IL-10 wurde zusätzlich analysiert, ob die Applikation der Testsubstanz die Immunantwort beeinflusste.

\section{Material und Methoden}

Hautmodelle. Die verwendeten Hautmodelle (4 mm epidermale Verwundung) wurden bei der MatTek Corporation (200 Homer Ave, Ashland, USA) erworben. Die Wundheilung wurde über einen Zeitraum von 5 Tagen (Tag 2 bis 6) untersucht. Es wurden je Zeitpunkt 5 den Tumor-Nekrose-Faktor- $\alpha$ (TNF- $\alpha$ ) sowie Wachstumsfaktoren (WF), unter anderem den Keratinozyten- (KGF) und den epidermalen Wachstumsfaktor (EGF), wodurch Keratinozyten und Fibroblasten chemotaktisch angelockt werden. In der Granulations- bzw. Proliferationsphase regen die sezernierten Zytokine und WFs die Migration und Proliferation von Bindegewebs- und Epi-

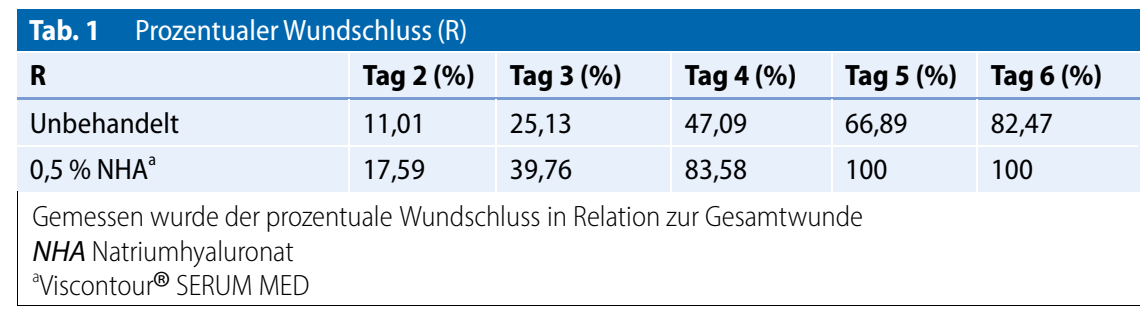




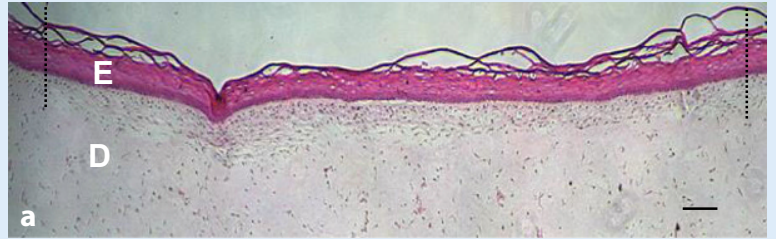

Epidermale Verwundung mittels $4 \mathrm{~mm}$ Biopsiestanze

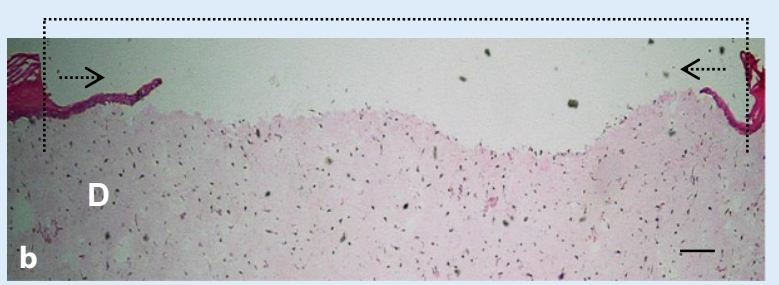

Abb. $1<$ Hämatoxylin-Eosin (H\&E)-Färbung eines 3-D-Vollhautmodells. a Natives, unverwundetes 3-D-Vollhautmodell. b 3-D-Hautmodell nach Entfernung der epidermalen Komponente. E Epidermis, D Dermis. Schwarze Pfeile: Migrationsrichtung der Keratinozyten zur Wundmitte. Messbalken $100 \mu \mathrm{m}$ unbehandelte und behandelte Modelle verwendet.

Testsubstanz. Als Testsubstanz kam Viscontour $^{\circledR}$ SERUM MED, Typ: Hydrogel, Applikation 2-mal täglich $10 \mu \mathrm{l}$, zum Einsatz.

Wirkstoff. Der Wirkstoff ist 0,5\% NHA fermentativen Ursprungs, frei von tierischen Eiweißen.

Trägerstoffe. Bei den Trägerstoffen handelt es sich um Wasser, Natriumchlorid, Natriumdihydrogenphosphat, Dinatriumphosphat.

NHA ist das Natriumsalz der Hyaluronsäure, das medizinische Anwendung findet.

In Vorversuchen konnte zwischen $10 \mu \mathrm{l}$ - und $20 \mu \mathrm{l}$-Applikation der Testsubstanz kein Unterschied bezüglich der Wundheilungsgeschwindigkeit festgestellt werden (Daten nicht gezeigt). Daher wurden $10 \mu \mathrm{l}$ verwendet.

Histologie. Die Modelle wurden für $24 \mathrm{~h}$ in $4 \%$ gepuffertem Formalin fixiert, im Tissue-Tek VIP entwässert (Sacura, Staufen, Deutschland) in Paraffin eingebettet, in $4 \mu \mathrm{m}$ starke Sektionen geschnitten (SM 200R Schlittenmikrotom, Leica, Wetzlar, Deutschland) und abschließend Hämatoxylin-Eosin (H\&E) (Autostainer Link48, Dako, Santa Clara, USA) gefärbt.

Reepithelisierungskinetik. Die Vermessung der Reepithelisierung wurde mit

\section{Ergebnisse}

\section{Verwundung}

Um eine Gewebeverletzung nachzustellen und die Reepithelisierungskinetik während der Wundheilung messen zu können, wurden die 3-D-Hautmodelle mit einer $4 \mathrm{~mm}$ Biopsiestanze epidermal verwundet (•Abb. 1b). Die - Abb. 1a zeigt exemplarisch ein H\&E-gefärbtes unverwundetes 3-D-Vollhautmodell. Dieses weist alle physiologischen Schichten nativen humanen Gewebes auf. Die - Abb. 1b zeigt ein Modell nach Entfernung der Epidermis. Zu erkennen ist die bereits beginnende Reepithelisierung des Gewebes durch die Migration der Keratinozyten von den Wundrändern hin zur Wundmitte (• Abb. 1 schwarze Pfeile).

\section{Wundheilung}

Je 5 Hautmodelle pro Zeitpunkt wurden mit der Testsubstanz $10 \mu \mathrm{l} \quad 0,5 \%$ NHA 2-mal täglich im Abstand von $8 \mathrm{~h}$ topisch behandelt. Die Wundheilungskinetik wurde täglich evaluiert, indem die Reepithelisierung anhand von H\&Egefärbten Paraffinschnitten mikroskopisch vermessen wurde (• Abb. 2, $\mathrm{L}_{\mathrm{REF}}$ ). Die Werte aller pro Zeitpunkt verwendeten Modelle wurden addiert, und der durchschnittliche Gesamtwundschluss in Mikrometer (-Abb. 3 ) sowie prozentual (-Tab. 1) wurde über den Beobachtungszeitraum von 5 Tagen (Tag 2 bis 6) analysiert. Der Wundschluss der unbehandelten Modelle (• Abb. 2a nach 2 Tagen, $\bullet$ Abb. 2 c nach 5 Tagen) sowie im Vergleich die mit der Testsubstanz behandelten Modelle (• Abb. 2b nach 2 Tagen, - Abb. 2d nach 5 Tagen) werden exemplarisch in $\bullet$ Abb. 2 dargestellt. Die mit 0,5\% NHA behandelten Modelle zeigten eine sowohl nach 2 als auch 5 Tagen deutlich schnellere Reepithelisierung als die unbehandelten Modelle. Nach 5 Tagen wiesen die unbehandelten Modelle einen durchschnittlichen Wundschluss von $66,89 \%$, bezogen auf die Gesamtwunde, auf. Die behandelten Modelle zeigten nach 5 Tagen bereits eine vollständige Reepithelisierung der Wunde sowie die beginnende Ausbil- 
dung einer Multilayer-Neoepidermis. Über den gesamten Beobachtungszeitraum (Tag 2 bis 6) konnte eine signifikant schnellere Reepithelisierungskinetik der behandelten Modelle objektiviert werden $(p<0,001$, • Abb. 3$)$.

\section{Immunantwort}

Zusätzlich zur Analyse der Reepithelisierungskinetik wurde täglich (Tag 2 bis 6) die Konzentration des antiinflammatorischen Markers IL-10 sowie des proinflammatorischen Zytokins IL-1 $\alpha$ mittels ELISA quantifiziert. Bereits an Tag 2 konnte eine erhöhte Expression von IL$1 \alpha$ in den mit $0,5 \%$ NHA behandelten Modellen nachgewiesen werden, die sich bis Tag 4 der Wundheilung signifikant $(p<0,001)$ von den unbehandelten Modellen unterschied (• Abb. 4). Parallel konnte eine signifikant $(p<0,001)$ geringere IL-10-Konzentration in den behandelten Modellen, verglichen mit den unbehandelten, bis einschließlich Tag 4 der Wundheilung nachgewiesen werden (• Abb. 5).

\section{Diskussion}

Die Daten der Wundheilungsexperimente zeigen, dass NHA die Reepithelisierung in einem epidermal verwundeten humanen 3-D-Vollhautmodell beschleunigt. Die zeitgleichen Veränderungen der IL$1 \alpha$ - und IL-10-Spiegel legen den Schluss nahe, dass NHA seine Wirkung darüber vermittelt. So wurden in den behandelten Modellen signifikant niedrigere IL10-Werte zwischen Tag 2 und 4 gemessen. Für das Zytokin IL-10 wurde bereits beschrieben, dass es während der Wundheilung eine entscheidende Rolle spielt [9]. Einerseits beeinflusst es die Funktion und Differenzierung von Fibroblasten und unterdrückt eine zu starke Immunantwort durch Herunterregulation von wichtigen proinflammatorischen Mediatoren wie IL-1 und TNF- $\alpha$ [13], andererseits reguliert es die hyaluronreiche extrazelluläre Matrix, wodurch ein entzündungsarmes Milieu geschaffen wird, damit die Wunde regenerativ heilen kann [10]. Eming et al. [4] konnten sowohl einen schnelleren Wundschluss als auch eine stärkere Wundkontraktion

Hautarzt 2017·68:987-993 https://doi.org/10.1007/s00105-017-4054-9

(c) Der/die Autor(en) 2017. Dieser Artikel ist eine Open-Access-Publikation.

L. Rüther · L. Bolke · G. R. Schlippe · W. A. Voss

\section{Hyaluronan als Schlüssel zur schnelleren Wundheilung in humanen 3-D-Vollhautmodellen}

\section{Zusammenfassung}

Hintergrund. Die Wundheilung kann in 3 Phasen unterteilt werden. 1) Exsudationsphase, 2) Granulationsphase, 3) Regenerationsphase. Der Regenerationsphase kommt besondere Bedeutung zu mit dem Ziel, die natürliche Barrierefunktion der Haut schnellstmöglich wiederherzustellen. Ziel der Studie war die Analyse der Reepithelisierungskinetik von unbehandelten sowie mit 0,5\% Natriumhyaluronat (NHA) behandelten humanen 3-DVollhautmodellen.

Methodik. Es wurden $10 \mu \mathrm{l}$ der Testsubstanz 2-mal täglich topisch appliziert. Die Analyse der Reepithelisierungskinetik erfolgte an den Tagen 2 bis 6. Die Beurteilung des Einflusses der Testsubstanz auf die Immunantwort erfolgte durch Quantifizierung von IL-1a und IL-10.

Ergebnisse. Die 2-mal tägliche Applikation von $0,5 \%$ NHA resultiert in einer erhöhten Reepithelisierungsgeschwindigkeit zu allen Zeitpunkten $(p<0,001)$. Diese Beobachtung geht einher mit einer geringeren Expression von IL-10 sowie einer erhöhten Expression von IL- $1 a$ an den Tagen 2 bis $4(p<0,001)$. Diskussion. Die Behandlung der humanen Hautmodelle mit NHA zeigte eine - verglichen mit unbehandelten Modellen - signifikant erhöhte Reepithelisierungsgeschwindigkeit des verwundeten Gewebes und führte zu einem schnelleren Wundschluss. Es liegt die Vermutung nahe, dass die Herunterregulation von IL-10 zu einer durch IL-1a mediierten, erhöhten Immunantwort führt und in der schnelleren Wundheilung resultiert. Folgestudien werden zeigen, ob die schnellere Wundheilung sowie die Modulation der Immunantwort durch die Applikation von $\mathrm{NHA}$ auch in vivo belegt werden kann.

Schlüsselwörter

Natriumhyaluronat · Inflammation . Reepithelisierung - Immunantwort . Wundschluss

\section{Hyaluronan as a key for accelerated wound healing in human 3D full thickness skin models}

\section{Abstract}

Background. Wound healing can be divided into three phases: (1) exsudation phase, (2) granulation phase, (3) regeneration phase. In particular, the epithelization phase is of great importance in order to quickly reconstitute the natural skin barrier. The aim of the present study was to determine the reepithelization kinetics of untreated and $0.5 \%$ sodium hyaluronate $(\mathrm{NHA})$ treated human 3D full thickness skin models. Materials and methods. The test protocol consisted of topically applying $10 \mu$ l of the test substance $0.5 \% \mathrm{NHA}$ twice a day. Evaluation of reepithelialization kinetics was carried out from days 2-6. Determination of the influence on immune response was performed based on quantification of IL-1a and IL-10.

Results. Application of $0.5 \%$ NHA twice a day enhanced the reepithelialization speed at all time points $(p<0.001)$. This observation is accompanied by a reduced expression of IL-
10 paralleled by an elevated expression of IL1 a on days $2-4(p<0.001)$.

Discussion. The treatment of human skin models with NHA resulted in a significantly increased reepithelization velocity of wounded tissue and consequently promoted faster wound closure, compared to untreated controls. It can be assumed that the downregulation of IL-10 caused the IL 1- $a$ mediated increased immune response which finally leads to accelerated wound healing. Follow-up studies will reveal if the faster wound healing and the modulation of the immune response through the application of $\mathrm{NHA}$ is valid in vivo.

Keywords

Sodium hyaluronate - Inflammation . Reepithelialization - Immune response . Wound closure 


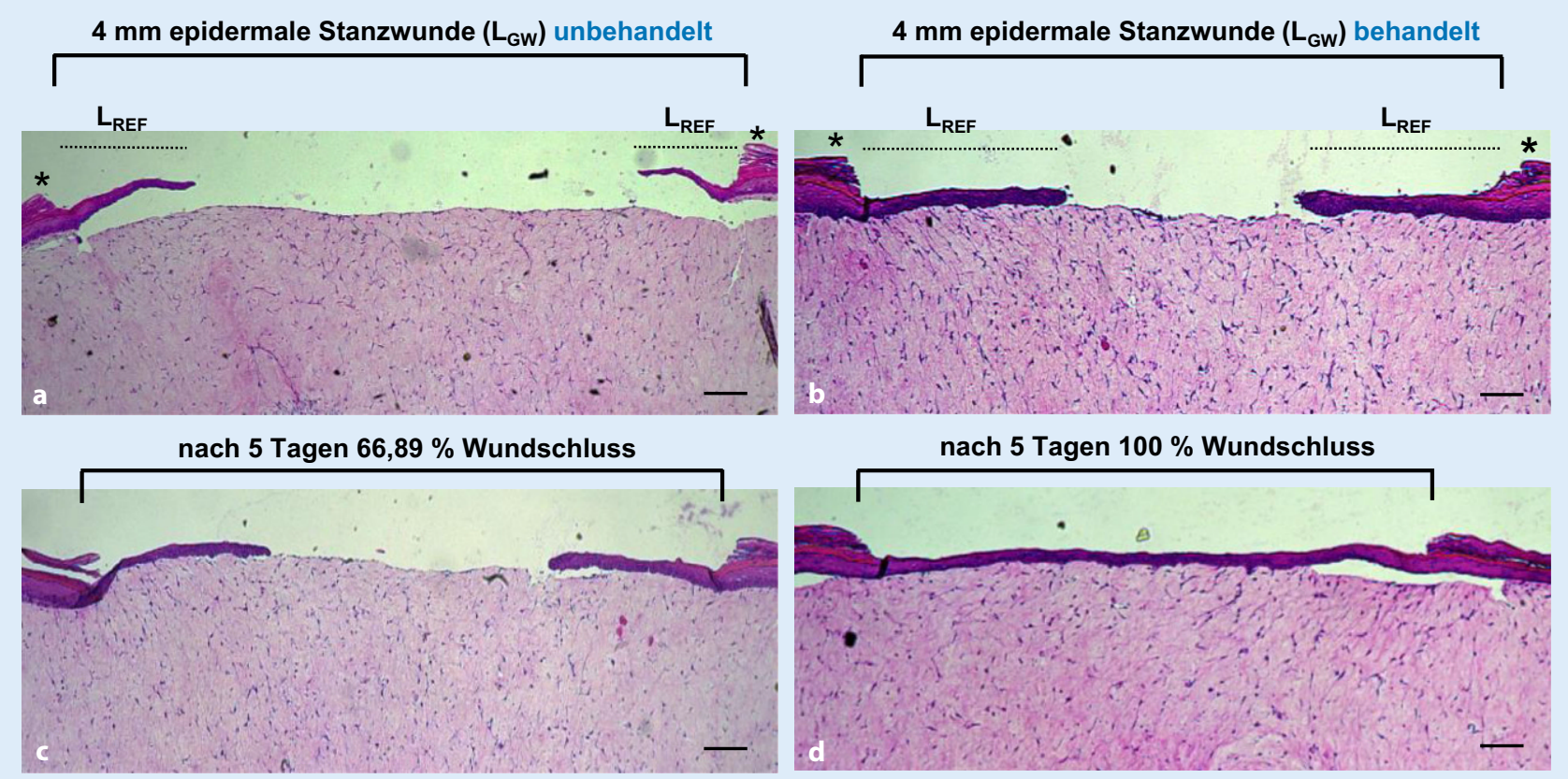

Abb. 2 ॥ Visualisierung der Migrationskinetik der Keratinozyten nach epidermaler Verwundung nach 2 und 5 Tagen mittels Hämatoxylin-Eosin (H\&E)-Färbung. a, c Wundschluss der unbehandelten 3-D-Modelle nach a 2 und $\mathbf{c} 5$ Tagen. b, d Wundschluss der mit 0,5\% Natriumhyaluronat behandelten 3-D-Hautmodelle nach b 2 und d 5 Tagen. $L_{R E F}$ Länge der Reepithelisierung (links und rechts), $L_{G W}$ Länge der Gesamtwunde. Messbalken $100 \mu \mathrm{m}$

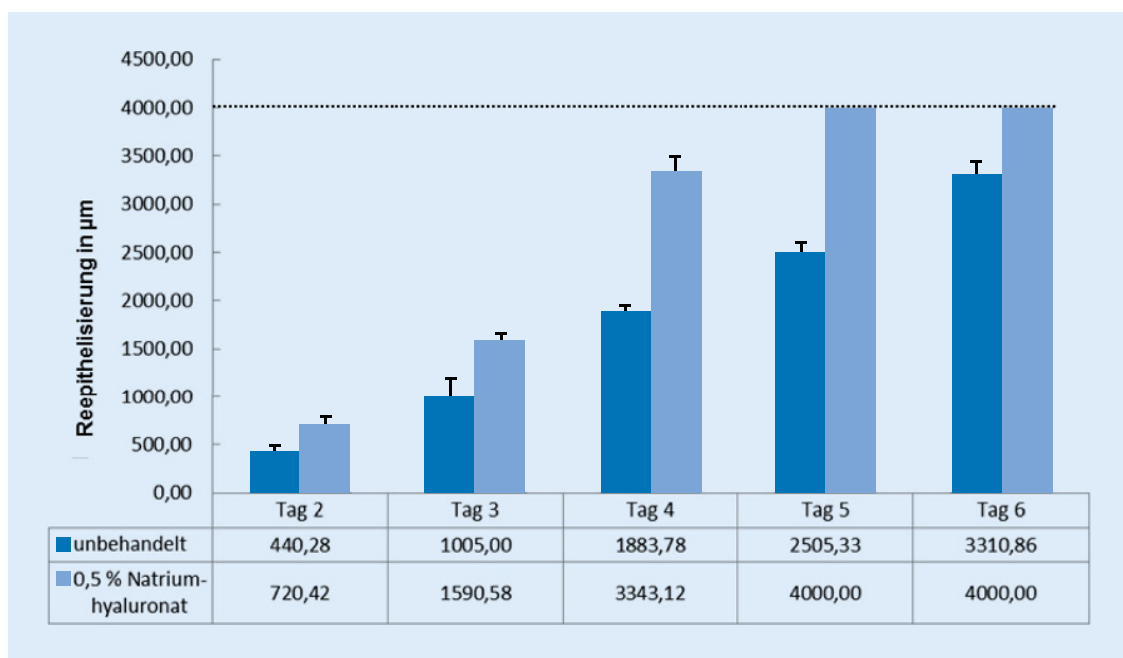

Abb. $3 \Delta$ Gesamtübersicht des Reepithelisierungsverlaufs (Tag 2 bis 6). Angaben in $\mu$ m. Gestrichelte Linie: vollständiger Wundschluss ( $4000 \mu \mathrm{m}$ Wunde)

in IL-10-defizienten Mäusen nachweisen, was die Vermutung nahelegt, dass IL-10 die Wundheilung „behindert“ oder zumindest bedeutend verlangsamt.

Gepaart mit der verringerten Expression von IL-10 wurde in den mit NHA behandelten Modellen eine erhöhte Konzentration des Zytokins IL-1 $\alpha$, einem Schlüsselfaktor für die Immunreaktion, beobachtet. Das proinflamma- torische Zytokin IL-1 $\alpha$ wird während der Wundheilung von Fibroblasten und Keratinozyten sezerniert und stimuliert seinerseits nachgeschaltete Mediatorensysteme wie IL-6 und IL-8. Zusammen mit weiteren proinflammatorischen $\mathrm{Zy}$ tokinen, wie beispielsweise TNF- $\alpha$, reguliert es zahlreiche Funktionen während der Wundheilung. So stimuliert IL-1 $\alpha$ die Proliferation von Fibroblasten und
Keratinozyten, orchestriert die Synthese und Degradation von extrazellulären Matrixproteinen und ist in die Fibroblastenchemotaxis involviert [21]. Durch die ausgeprägtere Inflammation wird offenbar die Wundheilung beschleunigt. Diese Hypothese wird durch die Ergebnisse von Singh et al. [17] untermauert, die eine schnellere Wundheilung durch eine erhöhte Expression von IL-1 $\alpha$ in einem Nagetiermodell beschrieben. Auch in der vorliegenden Studie scheint das applizierte NHA die Immunreaktion $\mathrm{zu}$ stimulieren, was letztlich in dem schnelleren Wundschluss resultiert.

\section{》) NHA beschleunigt die Reepithelisierung in einem epidermal verwundeten humanen 3-D-Vollhautmodell}

HA wird bereits in der operativen $\mathrm{Me}$ dizin eingesetzt, um eine bessere $\mathrm{Ab}$ heilung und Regeneration von chirurgischen Wunden zu gewährleisten [19]. Bei schlechtheilenden, chronischen Wunden zeigte eine klinische Studie an $125 \mathrm{~Pa}$ - 


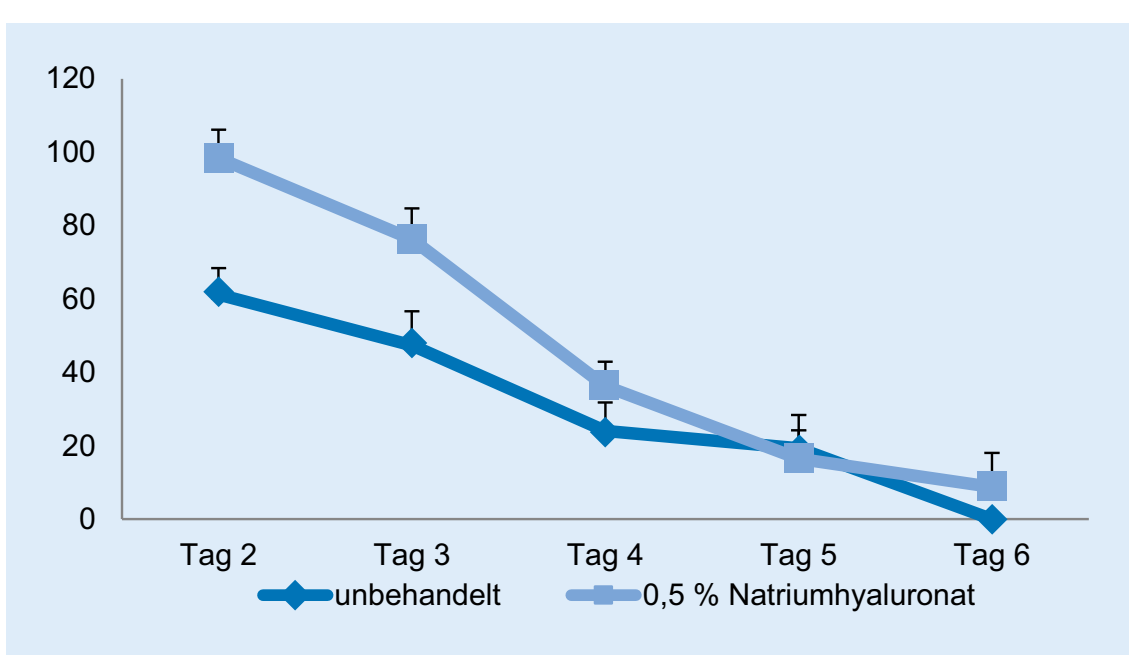

Abb. 4 A Konzentrationsverlauf des proinflammatorischen Zytokins Interleukin-1a

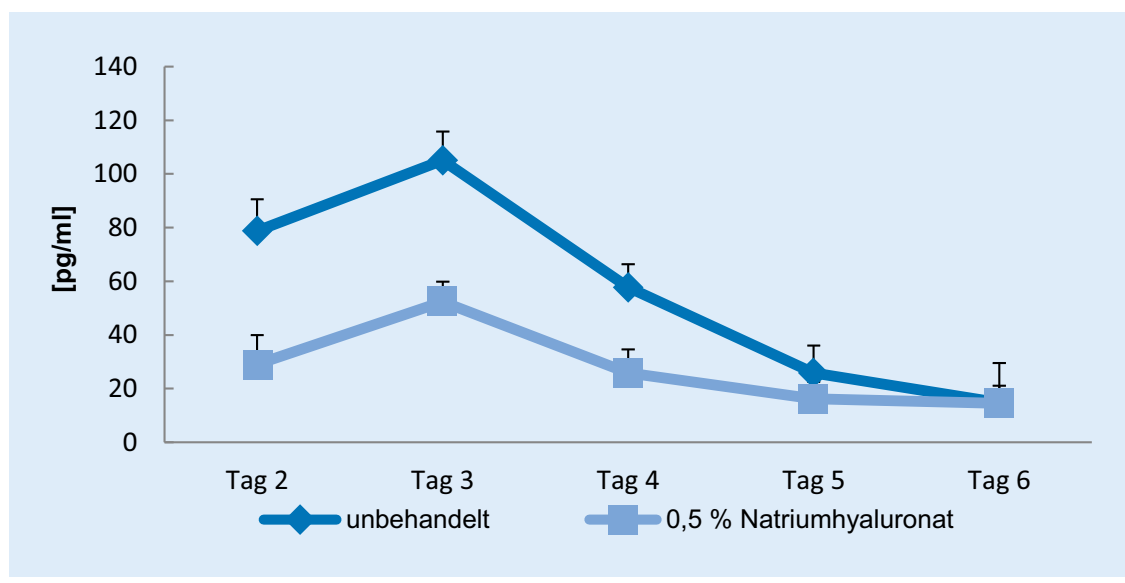

Abb. 5 - Konzentrationsverlauf des antiinflammatorischen Zytokins Interleukin-10

tienten mit Ulcus cruris eine tendenziell effektivere Wundheilung in der Patientengruppe, die mit einer Kombination aus HA und einem Hydrokolloidverband behandelt wurden, verglichen mit der Gruppe, bei der lediglich der Hydrokolloidverband Anwendung fand [11]. Weitere Belege hierfür stammen aus einer kontrollierten, randomisierten Doppelblindstudie mit 101 Patienten. Hier zeigten sich eine signifikante Reduktion des Wundareals der Ulzera sowie eine geringere Schmerzintensität der Wunde in der mit HA behandelten Gruppe [3].

Darüber hinaus sind bereits Ansätze publiziert worden, in denen HA als Kombinationspräparat in der Therapie von problematischen, chronischen Wunden erfolgreich eingesetzt wurde. So konnten Sobotka et al. [18] in einer klinischen Studie mit Patienten mit Ulcus cruris zeigen, diesen Wundtypus signifikant schneller heilen kann als herkömmliche Standardtherapien [20].

Auch für das in der vorliegenden Studie verwendete Produkt gibt es bereits erste klinische Daten aus einer unkontrollierten, monozentrischen Anwendungsbeobachtung. Untersucht wurden 3 Kollektive mit insgesamt 41 Patienten: 15 Patienten mit Ulcus cruris mit schlechter Heilungstendenz, 16 Patienten mit Spalthautentnahme sowie 10 Patienten mit akuten Wunden (operativer Eingriff). Dabei zeigte sich, dass die Behandlung mit Viscontour ${ }^{\circledR}$ SERUM MED sehr gut vertragen wurde, zu Schmerzfreiheit oder deutlicher Schmerzlinderung führte und in einer guten Granulationssowie raschen Epithelisierungstendenz der Wunden resultierte (unveröffentlichte Daten). Kontrollierte und randomisierte Studien, die die Wirksamkeit der Viscontour ${ }^{\circledR}$ SERUM MED-Therapie im Vergleich $\mathrm{zu}$ anderen Wundtherapeutika (oder einem Placebohydrogel) untersuchen, stehen aber noch aus.

\section{) Hyaluronan kann eine effektive Unterstützung der Wundheilung bieten}

Aus den bisher veröffentlichten, klinisch relevanten Daten wird ersichtlich, dass HA eine effektive Unterstützung der Wundheilung bieten kann. Vor allem als Kombinationspräparat angewandt besteht Hoffnung auf ein effizientes Wundtherapeutikum, das in der Behandlung schlecht heilender sowie chronischer Wunden Einsatz finden könnte.

\section{Limitationen}

den bestätigt. HA und Iod scheinen einen synergistischen Effekt aufzuweisen und für ein breites Spektrum von Wundtypen die Heilung zu beschleunigen.

Eine Metaanalyse von placebokontrollierten Studien beschreibt zudem, dass HA bzw. dessen Derivate den Heilungsverlauf in Brandwunden, chirurgischen Wunden sowie chronischen Wunden gegenüber traditionellen Therapien oder Placeboapplikationen signifikant verbessern. Besonders bei Ulcera crurum konnte gezeigt werden, dass $\mathrm{HA}$
Die in der vorliegenden Studie erhobenen Daten spiegeln trotz der Verwendung von Vollhautmodellen, die aus primären humanen Zellen kultiviert werden und eine gutes Modell für viele biologische Vorgänge darstellen, In-vitro-Ergebnisse wider. Obwohl In-vitro-Modelle das Verständnis molekularer Mechanismen und physiologischer Prozesse in den letzten Jahren weit vorangetrieben haben, spielt im Falle der Wundheilung das Fehlen eines nativen Gefäßsystems sowie einer 
dynamischen Umgebung im Organismus selbst (alternativer Metabolismus) eine nicht zu unterschätzende Rolle. Weiterführende Analysen mit geeigneten In-vivo-Modellsystemen sowie klinische Studien sollten folgen, um die in dieser Studie beschriebenen Ergebnisse zu überprüfen.

\section{Ausblick}

Noch immer wird nach optimalen wundheilungsfördernden Substanzen gesucht. Viele Studien machen deutlich, dass heutzutage kommerziell erhältliche, humane 3-D-Vollhautmodelle sehr gut geeignet sind, komplexe biologische Prozesse, wie beispielsweise die Wundheilung, relativ naturgetreu abzubilden. Die Weiterentwicklung im Bereich des „tissue engineering “ könnte langfristig Tierversuche im Bereich der Dermatologie obsolet werden lassen.

Zahlreiche Veröffentlichungen haben bereits die Wirkungsweise und hohe Effizienz von HA bei der Wundheilung beschrieben. Im Falle von postoperativen Wunden wird HA genutzt, um beispielsweise nach ophthalmologischen Eingriffen die Wundheilung und Regeneration voranzutreiben. Hierbei wird HA vornehmlich als viskoelastisches Gel eingesetzt, welches das Endothel der Hornhaut vor Beschädigung schützt. Bei problematischen, schlecht heilenden und chronischen Wunden haben sich Kombinationspräparate aus HA und Iod oder HA und Gelatine bewährt [22] und zeigen sehr gute Ergebnisse in der vollständigen Abheilung der Wunden, respektive der deutlichen Verkleinerung des Wundareals.

\section{》) Kombinationspräparate aus Hyaluronan und lod oder Hyaluronan und Gelatine haben sich bewährt}

Generell kann die Aktivität der HA während des gesamten Wundheilungsprozesses, beginnend bei der Blutgerinnung bis hin zur Remodellierung des neuen Gewebes, nachgewiesen werden. Dennoch werden HA-basierende Produkte zur therapeutischen Unterstützung der
Wundheilung in Deutschland im Vergleich zu den USA noch immer relativ wenig eingesetzt. Besonders im Bereich der Behandlung chronischer Wunden scheinen jedoch Patienten von den vielfältigen Funktionen von HA zu profitieren. Mehrere Studien belegen, dass bei Patienten mit Ulcus cruris durch Anwendung einer Kombinationstherapie beispielsweise mit HA und Iod die Wundheilung deutlich vorangetrieben werden kann.

\section{Fazit für die Praxis}

\section{- Die Anwendung von NHA bietet nachweislich eine intensiv hydrierte Matrix und schafft eine wundhei- lungsfördernde Umgebung. Daher sollte HA als therapeutisches Mittel zur Unterstützung der schnelleren Wundheilung bei diversen Wundty- pen vermehrt in Betracht gezogen werden. \\ - Die bereits in der Literatur beschrie- benen als auch in der vorliegenden Studie erhobenen Daten nähren die Hoffnung auf ein effektives Wund- therapeutikum, jedoch müssen die hier vorgestellten Daten zunächst durch kontrollierte klinische Studien überprüft und belegt werden.}

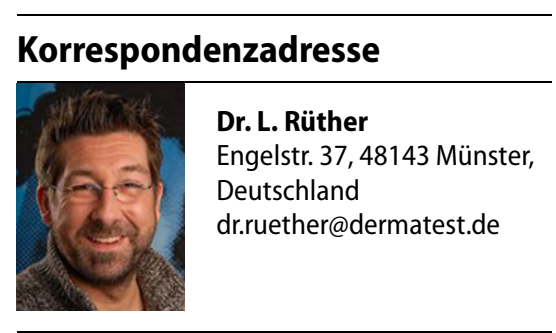

Förderung. Diese Veröffentlichung entstand im Rahmen einer bei der Dermatest ${ }^{\circledR} \mathrm{GmbH}$ mit der Testsubstanz durchgeführten In-vitro-Studie. Sponsor der Studie ist die Sanofi-Aventis Deutschland $\mathrm{GmbH}$, Berlin.

\section{Einhaltung ethischer Richtlinien}

Interessenkonflikt. L. Rüther, L. Bolke, G.R. Schlippe und W.A. Voss geben an, dass kein Interessenkonflikt besteht.

Die vorliegende In-vitro-Studie wurde an einem Hautmodell durchgeführt. Ein Ethik-Votum war nicht erforderlich.
Open Access. Dieser Artikel wird unter der Creative Commons Namensnennung 4.0 International Lizenz (http://creativecommons.org/licenses/by/4.0/deed. de) veröffentlicht, welche die Nutzung, Vervielfältigung, Bearbeitung, Verbreitung und Wiedergabe in jeglichem Medium und Format erlaubt, sofern Sie den/die ursprünglichen Autor(en) und die Quelle ordnungsgemäß nennen, einen Linkzur Creative Commons Lizenz beifügen und angeben, ob Änderungen vorgenommen wurden.

\section{Literatur}

1. Brenes RA, Ajemian MS, Macaron SH, Panait L, Dudrick SJ (2011) Initial experience using hyaluronate-iodine complex for wound healing. Am Surg 77(3):355-359

2. Chen WY, Abatangelo G (1999) Functions of hyaluronan in wound repair. Wound Repair Regen 7(2):79-89. https://doi.org/10.1046/j.1524-475X. 1999.00079.x

3. Dereure O,CzubekM,CombemaleP (2012) Efficacy and safety of hyaluronic acid in treatment of leg ulcers: a double-blind RCT. J Wound Care 21(3):131-2, 134-6, 138-9. https://doi.org/10. 12968/jowc.2012.21.3.131

4. Eming SA, Werner S, Bugnon P et al (2007) Accelerated wound closure in mice deficient for interleukin-10. Am J Pathol 170(1):188-202 https://doi.org/10.2353/ajpath.2007.060370

5. Gonzales AC, Costa TF, Andrade ZA, Medrado AR (2016) Wound healing - a literature review. An Bras Dermatol 91(5):614-620. https://doi.org/10. 1590/abd1806-4841.20164741

6. Hayden PJ, Bachelor M, Ayehunie S et al (2015) Application of MatTek in vitro reconstructed human skin models for safety, efficacy screening and basic preclinical research. Appl In Vitro Toxicol 1(3):226-233. https://doi.org/10.1089/aivt.2015. 0012

7. Hayden PJ, Petrali JP, Stolper G et al (2009) Microvesicating effects of sulfur mustard on an in vitro human skin model. Toxicol In Vitro 23(7):1396-1405. https://doi.org/10.1016/j.tiv. 2009.07.021

8. Kaya G, Rodriguez I, Jorcano JL, Vassalli $P$, Stamenkovic I (1997) Selective suppression of CD44 in keratinocytes of mice bearing an antisense CD44 transgene driven by a tissue-specific promoter disrupts hyaluronate metabolism in the skin and impairs keratinocyte proliferation. Genes Dev 11(8):996-1007.https://doi.org/10.1101/gad. 11.8 .996

9. King A, Balaji S, Le LD, Crombleholme TM, Keswani SG (2014) Regenerative wound healing: The role of interleukin-10. Adv Wound Care 3(4):315-323. https://doi.org/10.1089/wound.2013.0461

10. King A, Balaji S, Marsh E et al (2013) Interleukin10 regulates the fetal hyaluron-rich extracellular matrix via a STAT3-dependent mechanism. J Surg Res 184(1):617-617. https://doi.org/10.1016/j.jss. 2013.04.009

11. Meaume S, Ourabah Z, Romanelli M et al (2008) Efficacy and tolerance of a hydrocolloid dressing containing hyaluronic acid for the treatment of leg ulcers of venous or mixed origin. Curr Med Res Opin 24(10):2729-2739. https://doi.org/10.1185/ 03007990802367041

12. Oksala O, Salo T, Tammi R et al (1995) Expression of proteoglycans and hyaluronan during wound healing. J Histochem Cytochem 43(2):125-135

13. Peranteau WH, Zhang L, Muvarak N et al (2008) IL-10 overexpression decreases inflammatory 
mediators and promotes regenerative healing in an adult model of scar formation. J Invest Dermato 128(7):1852-1860. https://doi.org/10.1038/sj.jid. 5701232

14. Piatek S, Tautenhahn J (2012) Physiologie der Wundheilung. In: Lippert $\mathrm{H}$ (Hrsg) Wundatlas Kompendium der komplexen Wundbehandlung, 3. Aufl. Thieme, Stuttgart, S44-49

15. Safferling K, Sütterlin T, Westphal K et al (2013) Wound healing revised: a novel reepithelialization mechanism revealed by in vitro and in silico models. JCell Biol 203(4):691-709. https://doi.org/ 10.1083/jcb.201212020

16. Singer AJ, Clark RA (1999) Cutaneous wound healing. N Engl J Med 341(10):738-746. https:// doi.org/10.1056/NEJM199909023411006

17. Singh M, Nuulita K, Sinha I, Eriksson E et al (2016) Endotoxin-induced inflammation in a rodent model up-regulates IL-1a expression and CD45+ leukocyte recruitment and increases the rate of reepithelialization and wound closure. Wound Repair Regen 24(5):820-828. https://doi.org/10. 1111/wrr.12461

18. Sobotka L, Smahelova A, Pastronova J, Kusalova M (2007) A case report of the treatment of diabetic foot ulcers using a sodium hyaluronate and iodine complex. Int JLow Extrem Wounds 6(3):143-147

19. Valachova K, Volpi N, Stern R, Soltes L (2016) Hyaluronan in medical practice. Curr Med Chem 23(31):3607-3617. https://doi.org/10 2174/0929867323666160824162133

20. Voigt J, Driver VR (2012) Hyaluronic acid derivatives and theirhealing effect on burns, epithelial surgical wounds, and chronic wounds: a systematic review and meta-analysis of randomized controlled trials. Wound Repair Regen 20(3):317-331. https://doi. org/10.1111/j.1524-475X.2012.00777.x

21. Werner S, Grose R (2003) Regulation of wound healing by growth factors and cytokines. Physiol Rev 83(3):835-870

22. Wu S, Deng L, Hsia H et al (2017) Evaluation of gelatin-hyaluronic acid composite hydrogels for accelerating wound healing. J Biomater Appl 31(10):1380-1390

\section{Hier steht eine Anzeige.}

\section{算 Springer}

